\title{
вмJ Global Health Which men change in intimate partner violence prevention interventions? A trajectory analysis in Rwanda and South Africa
}

\author{
Andrew Gibbs (D) , ${ }^{1,2}$ Kristin Dunkle, ${ }^{1}$ Shibe Mhlongo, ${ }^{1}$ Esnat Chirwa, ${ }^{1,3}$
} Abigail Hatcher, ${ }^{3,4}$ Nicola J Christofides, ${ }^{3}$ Rachel Jewkes ${ }^{1,3,5}$

\begin{abstract}
To cite: Gibbs A, Dunkle K, Mhlongo S, et al. Which men change in intimate partner violence prevention interventions? A trajectory analysis in Rwanda and South Africa. BMJ Global Health 2020;5:e002199. doi:10.1136/ bmjgh-2019-002199
\end{abstract}

Handling editor Seye Abimbola

- Additional material is published online only. To view please visit the journal online (http://dx.doi.org/10.1136/ bmjgh-2019-002199).

Received 1 December 2019 Revised 21 February 2020 Accepted 7 March 2020

Check for updates

C Author(s) (or their employer(s)) 2020. Re-use permitted under CC BY-NC. No commercial re-use. See rights and permissions. Published by BMJ.

For numbered affiliations see end of article.

Correspondence to

Andrew Gibbs;

Andrew.Gibbs@mrc.ac.za

\section{ABSTRACT}

Introduction Emerging evidence suggests working with men to prevent intimate partner violence (IPV) perpetration can be effective. However, it is unknown whether all men benefit equally, or whether different groups of men respond differentially to interventions. Methods We conducted trajectory modelling using longitudinal data from men enrolled in intervention arms of three IPV trials in South Africa and Rwanda to identify trajectories of IPV perpetration. We then use multinomial regression to describe baseline characteristics associated with group allocation.

Results In South Africa, the Stepping Stones and Creating Futures (SS-CF) trial had 289 men and the CHANGE trial had 803 men, and in Rwanda, Indashyikirwa had 821 men. We identified three trajectories of IPV perpetration: a low-flat $(60 \%-67 \%$ of men), high with large reduction $(19 \%-24 \%)$ and high with slight increase (10\%-21\%). Baseline factors associated men in high-start IPV trajectories, compared with low-flat trajectory, varied by study, but included higher poverty, poorer mental health, greater substance use, younger age and more childhood traumas. Attitudes supportive of IPV were consistently associated with high-start trajectories. In separate models comparing high-reducing to high-increasing trajectories, baseline factors associated with reduced IPV perpetration were depressive symptoms (relative risk ratio, $\mathrm{RRR}=3.06$, $p=0.01$ SS-CF); living separately from their partner (RRR=2.14, $p=0.01$ CHANGE); recent employment (RRR=1.85, $p=0.04$ CHANGE) and lower acceptability of IPV (RRR=0.60, $p=0.08$ Indashyikirwa). Older aged men had a trend towards reducing IPV perpetration in CHANGE $(p=0.06)$ and younger men in Indashyikirwa $(p=0.07)$.

Conclusions Three distinct groups of men differed in their response to IPV prevention interventions. Baseline characteristics of past traumas and current poverty, mental health and gender beliefs predicted trajectory group allocation. The analysis may inform targeting of interventions towards those who have propensity to change or guide how contextual factors may alter intervention effects.

Trial registration numbers NCT03022370; NCT02823288; NCT03477877.

\section{Key questions}

What is already known?

- Interventions working with men and boys have the potential to prevent their perpetration of intimate partner violence (IPV).

- Little is known about which men benefit from ex isting interventions, since extant literature tends to group all participants into a single group.

What are the new findings?

- Using longitudinal data from three trials in South Africa (two trials) and Rwanda, we examined perpetration of IPV over 2 years of follow-up among those enrolled in the intervention arms.

- We identified three trajectories: a low-flat (with $60 \%-68 \%$ of men), high with large reduction $(19 \%-23 \%$ of men) and high with slight increase $(10 \%-21 \%)$.

- Those in the high-starting trajectories reported greater poverty, more childhood traumas, poorer mental health, higher substance misuse and attitudes supportive of IPV.

- Factors differentiating men in the high large reduction trajectory from those in the high slight increase trajectory included more depressive symptoms, older age, recent employment and less accepting of attitudes towards IPV.

What do the new findings imply?

- Working with men to prevent IPV has differential impact for different men, and targeting of interventions may bolster intervention effects.

\section{BACKGROUND}

Well-designed interventions to prevent men's perpetration of intimate partner violence (IPV) against their female partners are increasingly recognised as being potentially effective. ${ }^{1-3}$ They commonly aim to reduce the acceptability of violence, improve communication and relationship skills, encourage men to reflect on gender power and attitudes, and adopt more equitable behaviours, often termed as 
'transforming masculinities'. ${ }^{45}$ Some programmes also include aspects of livelihoods strengthening, while others are focused more broadly around improving father-child relationships, or within a sexual and reproductive health framework (eg, Doyle et al and Ruane-McAteer et $a l^{67}$ ) and are often implemented alongside women.

Two broad approaches to working with men on IPV prevention have been dominant. The first are small group participatory interventions working with men (and sometimes their female partners) intensively over many hours (often 40-50 hours). ${ }^{1}$ These interventions provide ongoing spaces for men to reflect on their behaviour and the underlying drivers of this. ${ }^{158}$ The second dominant approach is community social norms transformation led by activists over a longer period (usually 18-24 months), trying to reduce social acceptance of violence, and in particular IPV, and gender inequality. ${ }^{9}$ These include community wide campaigns, highlighting violence as a problem and challenging its use, alongside small group sessions. Critically, they seek to impact whole communities, rather than just those directly targeted by the intervention.

As the evidence-base around IPV prevention interventions working with men has grown, it is important for the field to ask more penetrating questions such as among men who to participate in IPV prevention interventions, how does their use of IPV change over time? And, closely linked, are certain men more likely to change, and if so, what are characteristics of men who do?

Existing research highlights how different constructions of masculinity have varying relationships to men's use of violence in intimate relationships. ${ }^{10}{ }^{11}$ In any given setting, there are multiple masculinities, shaped by experiences of poverty, gender norms and abuse in childhood. ${ }^{12}$ In South Africa, Jewkes and Morrell ${ }^{13}$ used population based quantitative data to describe three different masculinity groups and their use of violence. One group (a quarter of the sample) used IPV extensively, a second group (30\%) used IPV less so, but still a lot and then a third group ( $46 \%$ of the sample) used IPV relatively infrequently. Men who were in the most violent group were more likely to have poor mental health, greater childhood trauma exposure, more gender inequitable attitudes and engage in other antisocial practices such as having an illegal gun, and crime, more than other men. ${ }^{13}$ These may all influence men's engagement and ability to change through structured interventions, although it is unclear exactly how and to what extent this depends on intervention content or surrounding context.

Other research has sought to describe patterns around changing men. In qualitative research, Gibbs $e t$ $a l^{4}$ described three groups of men who participated in a gender transformative and livelihoods intervention. For one group, the intervention had no effect, a second group described trying to change while the intervention was ongoing, but once it had finished lapsing back to previous behaviours. A third group described positive change, and 'growing up', essentially developing a new masculine position for themselves through distancing themselves from a more youthful identity. ${ }^{14}$ This work raises intriguing and important questions about what distinguished the men who described themselves as 'growing up' and specifically: whether there are specific baseline characteristics that could identify men predisposed to such transformation? It may also be that these observed patterns were artefacts of the qualitative study, and thus larger scale replication is warranted.

A second outstanding question is: to what extent do interventions lead to different responses by men, depending on intervention approaches or the context in which they are implemented? The most common intervention approaches, intensive group-based participatory approaches and 'lighter touch' community activism interventions, are distinct approaches with very different underlying theories of change, and may therefore impact differently on different men. For example, they may be more effective with some types of men than others or work differently in certain contexts depending on the prevailing cultural constructions of masculinities.

In this paper, we use data from evaluations of interventions which included men from two very different African countries. The first intervention, Stepping Stones and Creating Futures (SS-CF), was implemented with younger (18-30-year-old) men in urban informal settlements in South Africa and was an intensive gender transformative and livelihoods strengthening intervention. ${ }^{15}$ The second intervention, the Sonke CHANGE trial, was implemented in peri-urban settlements in South Africa, working with a wider age range (18-49 years) and comprised a social norms change intervention, which worked with community activists over 18 months. ${ }^{16}$ The third intervention, Indashyikirwa, was implemented in rural Rwanda and worked with married and cohabiting male-female couples aged 18-49 who were enrolled in village savings and loan associations (VSLA). It provided an intensive gender transformative intervention coordinated with a larger programme of community-level activities aimed to create an enabling environment for change. ${ }^{17}$

The aims of this paper are twofold. To describe the trajectories over time of men's perpetration of IPV among those in the intervention arm of each study, and to describe the baseline factors associated with men's allocation to each trajectory. To achieve this, we identify clusters of men based on their IPV perpetration over time using group-based trajectory modelling. ${ }^{18}$ We then assess the baseline characteristics associated with men's allocation into each trajectory, and assess differences between the groups.

\section{METHODS}

Data are drawn from men in the intervention arms of three cluster randomised controlled trials, which enrolled and tracked a cohort of individual male study 
participants and had assessments at baseline, 12 months and 24 months, with data on IPV collected using comparable measures. We use trajectory modelling and multinomial regression within each study to answer the aims. Below we describe the projects in detail.

\section{Stepping Stones and Creating Futures Intervention}

SS-CF trial, worked with young men and women living in urban informal settlements in eThekwini Municipality (Durban), South Africa. The intervention is group-based and participatory, and encouraged young people to strengthen their livelihoods and transform their gender relationships, through 21 sessions, each 3 hours long. ${ }^{15}$ The intervention was delivered by trained peer facilitators, with sessions twice a week.

\section{Study design}

We conducted a two-arm cluster randomised control trial. Clusters were informal settlements, with naturally occurring boundaries. In each cluster, we recruited $\sim 20$ men (and $\sim 20$ women) in conjunction with our NGO implementation partner, Project Empower. More information on study design is available. ${ }^{15}$

\section{Main IPV trial outcomes}

In the main analysis, men in the SS-CF intervention reported significantly less physical IPV (adjusted ORs $(\mathrm{aOR}) 0.71,95 \%$ CI: 0.51 to $0.97, \mathrm{p}=0.032$ ), and a marginal reduction in sexual IPV (aOR0.74, 95\% CI: 0.54 to 1.03, $\mathrm{p}=0.072$ ) perpetration at 24 months, with similar levels of reduction seen at 12 months. ${ }^{19}$

\section{Sonke CHANGE trial}

\section{Intervention}

The Sonke CHANGE trial worked with men (18-40 years) in peri-urban settlements in Gauteng, South Africa, to reduce IPV through community activism and mobilisation approaches to transforming harmful gender attitudes and norms. The intervention comprised door-to-door activities, 2-day workshops drawing on the Sonke CHANGE curriculum, which sought to encourage reflection around gender norms and the use of violence, mini-workshops (3-4hours), community murals and deploying community action teams over 18 months.

\section{Study design}

A two-arm cluster randomised control trial. Clusters were demarcated areas within one large peri-urban settlement. Approximately 120-150 men were recruited in each cluster. More information on study design is available. ${ }^{16}$

\section{Main outcomes for IPV}

In the main analysis, men in the CHANGE trial showed no difference in the cluster-level proportion past year physical IPV (diff $=0.002,95 \%$ CI $=-0.07-0.08$ ) or sexual IPV (diff $=0.01,95 \% \mathrm{CI}=-0.04-0.063)$ at endline after adjusting for sociodemographic characteristics and baseline IPV perpetration. ${ }^{20}$ Severe IPV followed a similar pattern (diff $=0.01 ; 95 \% \mathrm{CI}=-0.05-0.07)$.

\section{Indashyikirwa}

Intervention

Indashyikirwa was an intensive gender transformative and relationship strengthening intervention working with married or cohabiting couples enrolled in VSLA groups in Rwanda. The intervention had 21 sessions of 3 hours for couples, focused on gender transformation, managing common triggers of conflict and improved communication. The curriculum drew on components of previous promising interventions, including Journeys of Transformation, which was developed by CARE Rwanda, Promundo and RWAMREC (Rwanda Men's Resource Center) to foster men's support of women's economic empowerment and improve household relations. It also incorporated the frameworks for understanding different types of interpersonal and intrapersonal power developed by the SASA! programme in Uganda. ${ }^{21} 22$

\section{Study design}

A two-arm cluster-randomised control trial comparing the couples receiving the intervention, to those participating only in the VSLA. Couples were either married and/or cohabiting and one (or both) were active in the VSLA. More information is available on the study design. ${ }^{23}$

\section{Main outcomes for IPV}

In the main analysis, men's perpetration of sexual IPV was significantly reduced at 24 months (adjusted risk ratio $(\mathrm{aRR})=0.52,95 \%$ CI: 0.37 to $0.74, \mathrm{p}<0.001)$, and there was a non-significant reduction in men's perpetration of physical IPV ( $a R R=0.79,95 \%$ CI: 0.56 to $1.09, p=0.15$ ). There was also an overall reduction in severe IPV perpetration $(\mathrm{aRR}=0.54,95 \% \mathrm{CI}: 0.38$ to $0.75 ; \mathrm{p}<0.001)$.

\section{Patient and public involvement}

Patients and the public were not involved in the design, implementation, analysis or dissemination of the study.

\section{Data collection}

All studies trained same-gender fieldworkers to collect data, and primarily used self-completed questionnaires on computers/cellphones, in relevant local languages. In the Sonke CHANGE trial and Indashyikirwa, audioassisted self-completed interviews were also used. In all studies, a face-to-face option for participants was also possible.

\section{Measures}

Past year physical and/or sexual IPV: in all three studies, men were asked behaviourally specific questions about physical (five items) and sexual (three items) IPV in the past year, using questions modified from the WHO's Violence Against Women (VAW) Scale,${ }^{24}$ as also tested in the UN multicountry Study on Men and Violence in Asia and the Pacific. ${ }^{25}$ An example item is: 'In the last 12 months how many times did you slap your current 
or previous girlfriend or wife or throw something at her which could hurt her?' Responses were 'never', 'once', 'few' and 'many'. The eight items were scored and summed together (range 0-24), to create a severity of physical and/or sexual IPV measure.

We assessed sociodemographic information at baseline. Specifically age and education level, which in South Africa we coded as only primary, secondary not complete, secondary completed and in Rwanda as either none or some primary or more. Relationship status was asked in a single item, and in South Africa coded as living with/ married to partner, having a partner but not living with her, and no current relationship, while in Rwanda as they were all couples, relationship status was coded as married legally versus living together.

Poverty was assessed using a range of items. Food insecurity in South Africa was assessed with a three-item scale about past month household food-insecurity ${ }^{26}$ and in Rwanda two of these items were used, and in all cases summed and treated as a score with higher scores denoting greater food insecurity. In the South African studies, there was a single item about stealing in the past month because of hunger. In all three countries, we asked a single item about earnings in the past month, and recoded this to none versus any. Finally, in South African studies, we asked whether men had worked in the past 3 months (no vs yes); this was not asked in rural Rwanda because the men were primary engaged in agricultural subsistence.

We also assessed gender attitudes and practices. In SS-CF, we asked five items comprising the relationship control scale (eg, I tell my partner who she can spend time with), with a four-point Likert Scale (strongly disagree to strongly agree: range $0-24$, Cronbach's alpha $0.67)$. Men were also asked about their attitudes towards VAW (eg, there are times when a woman deserves to be beaten) with responses on a four-point Likert Scale. In both South African studies, two-items were asked, and in Rwanda five items. Men were coded as having attitudes accepting of violence towards women if they answered agreed or strongly agreed to any item.

Men were asked about their mental health and alcohol use. In South Africa, the 10-item Alcohol Use Disorders Identification Test (AUDIT) scale was used, ${ }^{27}$ while in Rwanda the short version of this, AUDIT-C, was used, which mainly assessed heavy episodic drinking, with items summed in both (larger equals more alcohol use). Depressive symptoms were assessed using Centre for Epidemiologic Studies Depression (CES-D) scale $^{28}$ the full scale (20 items) was used in South Africa and a binary created (depression $\geq 21$ ), in Rwanda, a 10-item version of CES-D was used, with a binary created (depression $\geq 12$ ).

Finally, men's childhood trauma exposure was assessed. In all countries, a single item asked whether a man had witnessed his mother being beaten by a partner/husband (never vs sometimes/many times). In South Africa physical, sexual and emotional abuse and neglect were also assessed (11 items SS-CF 13 items Sonke CHANGE) using a modified questionnaire based on the on the short form of the Childhood Trauma Questionnaire, ${ }^{29}$ with responses never, sometimes, often or very often, which was summed to create a score (SS-CF: range 11-41; Sonke CHANGE: range 13-56, larger equates to more childhood trauma). In Rwanda, a single item assessed how often before the age of 18 a man was beaten (never and sometimes verses often/very often).

\section{Statistical analysis}

The analysis applied group-based trajectory models to identify groups/clusters of men with similar progression patterns of physical and/or sexual IPV scores over time. This method assumes that the population is composed of a mixture of distinct groups defined by the developmental trajectories. ${ }^{18}$ This approach uses the best fitting function to the outcome from a family of flexible polynomials and allows the prediction of each participant's probability of membership in each identified trajectory group. $^{18}$

Models with two, three and four trajectory groups were examined. The optimal number of trajectory groups for describing perpetration of IPV over time was based on describing a more realistic depiction of the possible trajectories that one can obtain and statistical goodness of fit statistics such as BIC and AIC. The final model used had three trajectory groups. Our model assumed one flat and two linear polynomials. Due to limited number of data points and to avoid overfitting, quadratic and cubic polynomials were not considered.

Only men with at least two data points were considered for analysis. The analysis was conducted using STATA V.15 using command, traj, to obtain probabilities of group membership for the three trajectory groups and membership to a trajectory was determined by the maximum posterior probability of group membership. The zero-inflated Poisson distribution was implemented for analysis, as we used scores for IPV perpetration.

To profile the baseline characteristics associated with each group/cluster, we assessed Pearson's $\chi^{2}$ test for categorical variables, and analysis of variance for continuous variables presenting number/means or percentages/ $\mathrm{SD}$ and p-values as appropriate. We then fitted multinomial multivariate regression models comparing the lowflat trajectory to the other trajectories. The final model was obtained through a backward hierarchical model building process which started with sociodemographic factors using 0.2 significance level for entry and 0.1 significance to remain. We then repeated the procedure adding the childhood violence exposure/experience factors, then followed by gender attitudes and relationship practices and lastly mental health status and substance abuse factors. We reported adjusted RRRs, 95\% CI and p-values for the final model.

We then assessed differences between the highincreasing trajectory and the high-reducing trajectory, using multinomial multivariable models. We used the 
same backward hierarchical model building as above, and report RRR, 95\% CI and p-values.

\section{RESULTS}

In total, 289 men in the intervention arm of SS-CF provided two or three time points, while this was 803 men in the intervention arm of Sonke CHANGE, and 806 in Indashyikirwa. Baseline descriptive data (table 1) show men's mean age in SS-CF was 23.9 years, compared with 27.2 years in Sonke CHANGE and 35.7 years in Indashyikira. In both South African studies, about a third had completed secondary school $(30.5 \%$ in SSCF; $38.2 \%$ in Sonke CHANGE), and in Indashyikirwa almost half $(49.0 \%)$ reported having completed primary education. In SS-CF, almost no men lived with their partners (7.3\%), and two-thirds reported a partner they did not live with $(67.8 \%)$. In contrast, a third (37.0\%) of Sonke CHANGE participants reported they lived with their partner, while half $(49.1 \%)$ reported they had a partner they did not live with. Indashyikirwa enrolled only men in established couples; two-thirds $(69.1 \%)$ were legally married, while a third $(30.9 \%)$ were cohabiting.

Men in all studies were poor, and in the past month a third (37.0\% in SS-CF and $30.4 \%$ in Sonke CHANGE) reported stealing because of hunger, while in SS-CF, $58.8 \%$ had earnings in the past month compared with three-quarters (76.6\%) in Sonke CHANGE and 72.8\% in Indashyikirwa. Beating of a partner was reported as acceptable by just under half the men in all studies (46.0\% in SS-CF; $43.1 \%$ in Sonke CHANGE; $41.0 \%$ Indashyikirwa). Many men reported witnessing their mother being beaten by her partner/husband while they grew up (33.7\% SS-CF; 25.9\% Sonke CHANGE; $44.8 \%$ Indashyikirwa). Depressive symptoms varied across the studies, ranging from $47.9 \%$ in SS-CF, $27.5 \%$ Sonke CHANGE and $12.7 \%$ in Indashyikirwa.

\section{Prevalence of IPV and trajectories of change}

Three group trajectories of men's perpetration of physical and/or sexual IPV were identified in the model through statistical fit and theoretical plausibility. The trajectories were (a) a low-flat trajectory of IPV perpetration, (b) a trajectory starting with high rates of perpetration and declining sharply at midline and endline to close to the low-trajectory and (c) a trajectory starting high and slightly increasing over the study period.

In SS-CF, the overall prevalence of physical and/or sexual IPV in the intervention arm was $55.6 \%$ at baseline, $52.7 \%$ at midline and $41.8 \%$ at endline. Within this overall pattern of decline, there were three discrete trajectories of severity of IPV perpetration: $60.4 \%$ were in the flat-low trajectory; a fifth (19.1\%) in the high-reducing trajectory and a fifth $(20.5 \%)$ in the high slight increasing trajectory (see figure 1, and online supplementary table 1 ).

In the Sonke CHANGE trial, there was a steep decrease in past year physical and/or sexual IPV in the intervention arm from $45.7 \%$ at baseline, to $39.0 \%$ midline and $26.9 \%$ at endline (though overall the intervention showed no impact because of similar patterns in the control $\mathrm{arm}^{20}$ ). Two-thirds $(66.0 \%)$ of men were in the flat-low trajectory, one-fifth $(19.1 \%)$ were in the high decreasing trajectory and $14.8 \%$ were in the high slightly increasing trajectory (figure 2 and online supplementary table 1 ).

In Indashyikirwa, men in the intervention group reporting two or three data-points, $37.1 \%$ reported any past year physical and/or sexual IPV at baseline 24.0\% at midline and $22.6 \%$ at endline. In terms of trajectories, two-thirds $(66.5 \%)$ were in the flat-low trajectory, a quarter $(23.8 \%)$ were in the reducing trajectory and $9.7 \%$ were in the high-increasing trajectory (figure 3 and online supplementary table 1 ).

\section{Factors associated with membership of each trajectory}

Descriptively, across the three studies, there was no clear association between membership in the different group trajectories for age, relationship status and education (table 1). In Sonke CHANGE, men in the two highstart point trajectories were younger $(p=0.012)$, while in Indashyikirwa, there was a suggestion that men in the high-reducing trajectory were younger than those in the high-increasing trajectory $(\mathrm{p}=0.107)$. For education, in Sonke CHANGE, a lower proportion in the hightrajectories reported completing high school (41.9\% low-flat; $28.2 \%$ high-reducing; $34.8 \%$ high-increasing, $\mathrm{p}=0.025$ ), compared with the low-flat trajectory. While for relationship status, in Sonke CHANGE, compared with the low-flat trajectory, men in the high-increasing trajectory were more likely to live with their partner $(33.7 \%$ low-flat; $39.7 \%$ high-reducing; $48.3 \%$ high-increasing). In SS-CF, there were no associations seen on these variables, and in Indashyikirwa, no association with education or relationship.

There were, however, many commonalities descriptively across the three studies around men in the highstart point trajectories, with those men reporting poorer mental health, greater substance misuse, more childhood traumas and more acceptance of wife beating, than men in the low-trajectories. In South Africa, higher poverty levels were also indicative of being in the higher start point trajectories.

In both South African studies, men in the two high-start point trajectories were poorer than those in the low-flat trajectory. In SS-CF, a greater proportion in the high-start point trajectories reported stealing in the past month because of hunger (low-flat: 27.7\%; high-reducing: 42.9\%; high-increasing: $55.2 \%, \mathrm{p}<0.001$ ), and they also reported a greater likelihood of earning anything in the past month (low-flat: $51.2 \%$; high-reducing: $66.1 \%$; highincreasing: $71.6 \%, \mathrm{p}=0.008)$. In Sonke CHANGE, men in the high-start point trajectories higher food insecurity scores compared with the low-flat trajectory $(\mathrm{p}=0.008)$, and a greater proportion reported stealing because of hunger (flat-low: 20.8\%; high-reducing: $51.9 \%$; high slight increase: $45.2 \%, \mathrm{p}<0.001)$. 


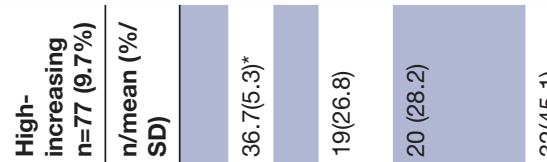

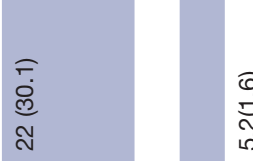

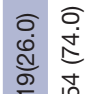

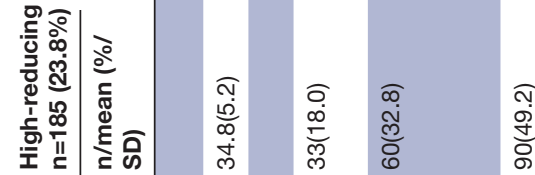

$\begin{array}{ll}\widehat{m} & \kappa \\ \dot{0} & 0 \\ 0 & 0 \\ \sigma & 0 \\ \sigma & 0\end{array}$

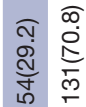

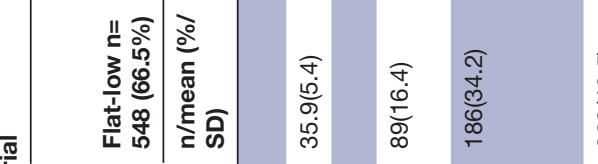

बृ.

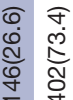

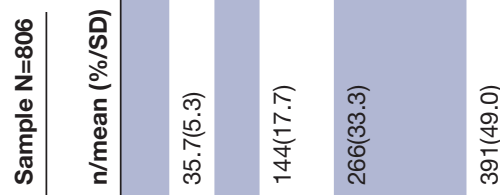

임

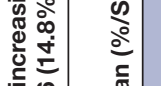

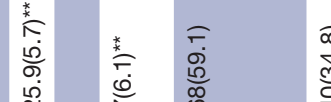

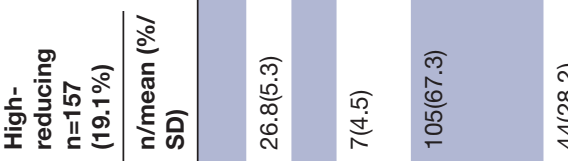

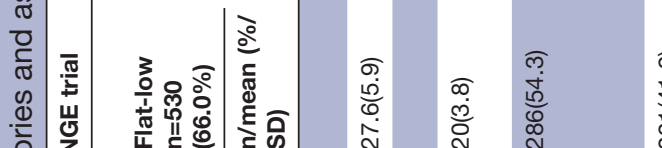

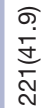

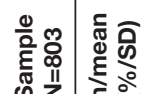

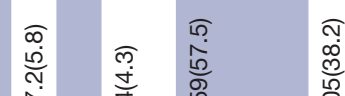

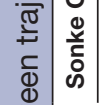

का

ก 年 每

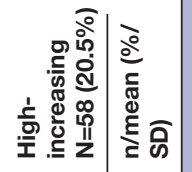

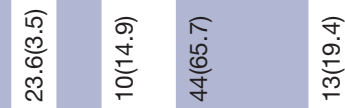

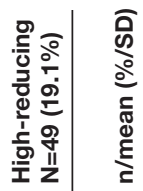

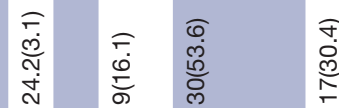

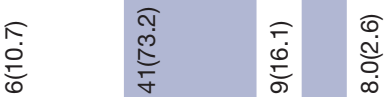

穴

क.

ธุก โุ

ำ

$\stackrel{\text { N }}{\frac{N}{\infty}}$

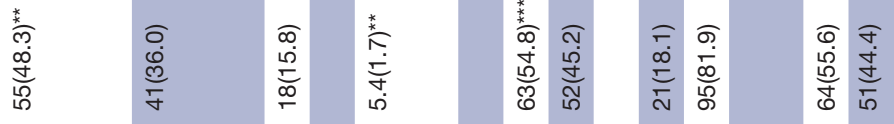

焉

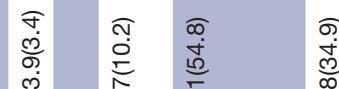

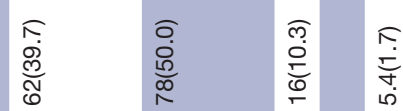

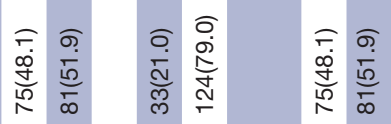

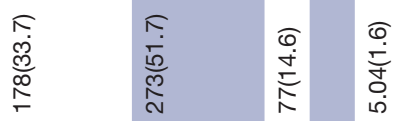

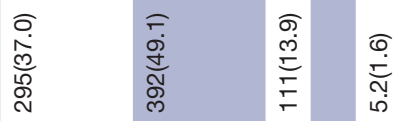

ब.

察

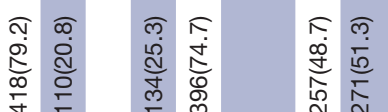

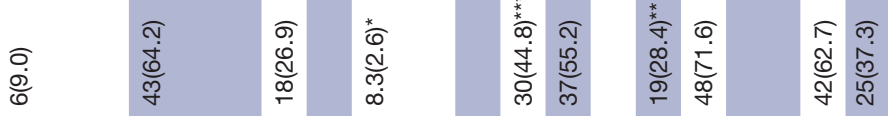

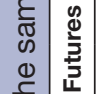

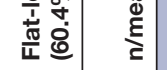

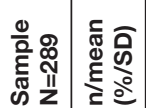

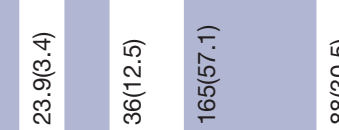

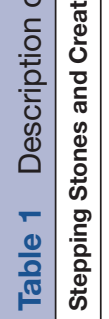

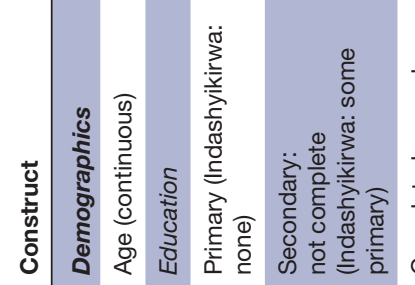

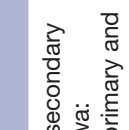

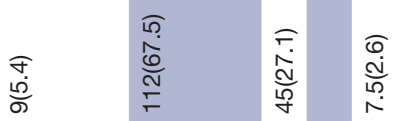

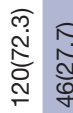

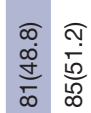

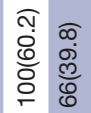

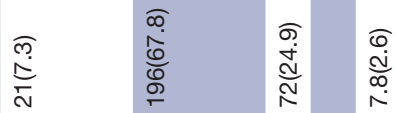

ธิ ธิ สิ

ल.

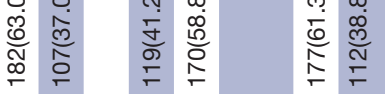




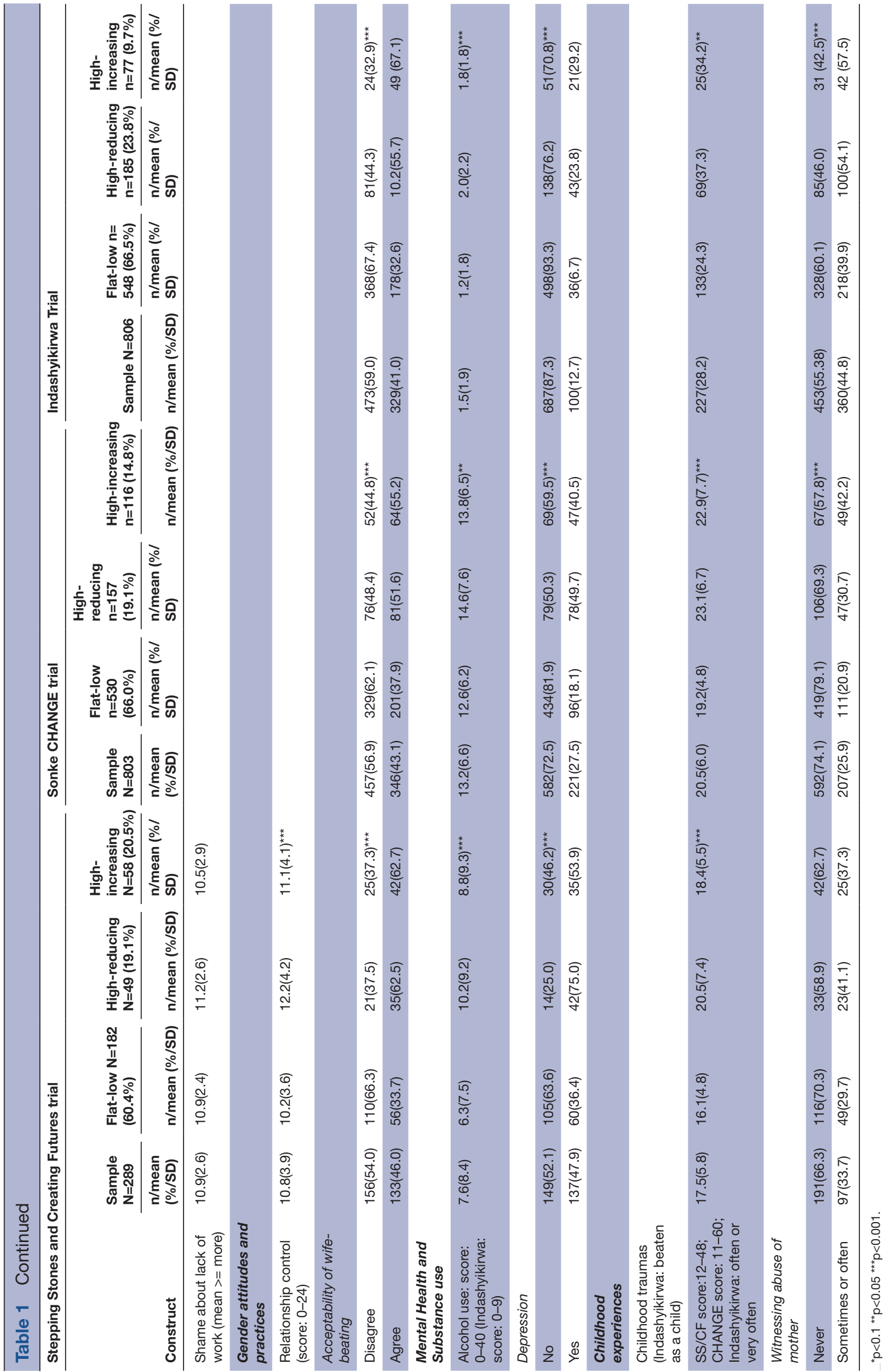

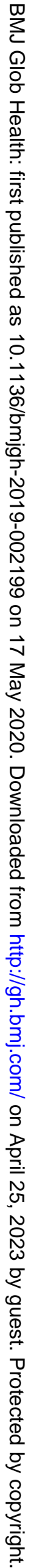




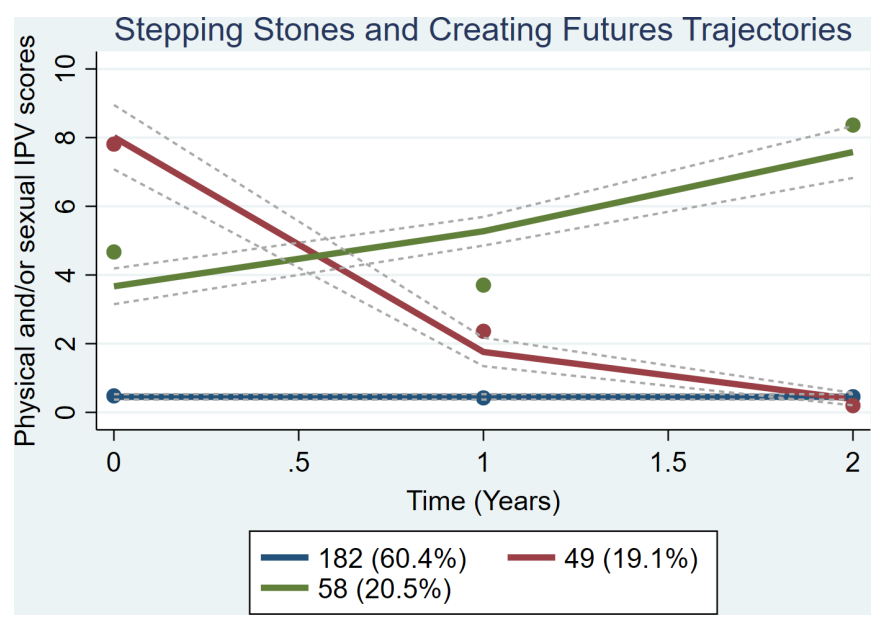

Figure 1 Trajectories of men's perpetration over time in the Stepping Stones and Creating Futures trial.IPV, intimate partner violence.

In all three studies, men in the high-start trajectories were more likely to report gender inequitable attitudes and practices. In SS-CF, men's mean scores for controlling behaviours were greater among those in the high trajectories, compared with the flat low trajectory (flat-low: 10.2; high-reducing: 12.2; high-increasing: 11.1, $\mathrm{p}<0.001$ ), and a greater proportion of men reported agreeing that wife beating was acceptable (low-flat: 33.7\%; high-reducing: $62.5 \%$; high-increasing: $62.7 \%$, $\mathrm{p}<0.001)$. In Sonke CHANGE, similarly greater proportion in the high-reducing (51.6\%) and high-increasing $(55.2 \%)$ trajectories endorsed agreement of wife beating, compared with the low-flat trajectory $(37.9 \%, \mathrm{p}=0.004)$, and greater endorsement of wife beating was also seen in Indashyikirwa (32.6\% low-flat; $55.7 \%$ high-reducing; $67.1 \%$ high-increasing, $\mathrm{p}<0.001)$.

Men in the high-start point trajectories across all three studies reported more experiences of childhood trauma. In SS-CF and Sonke CHANGE, mean scores were higher for overall childhood trauma $(p<0.001$ SS-CF; $p<0.001$ Sonke CHANGE), and in Indashyikirwa, a greater

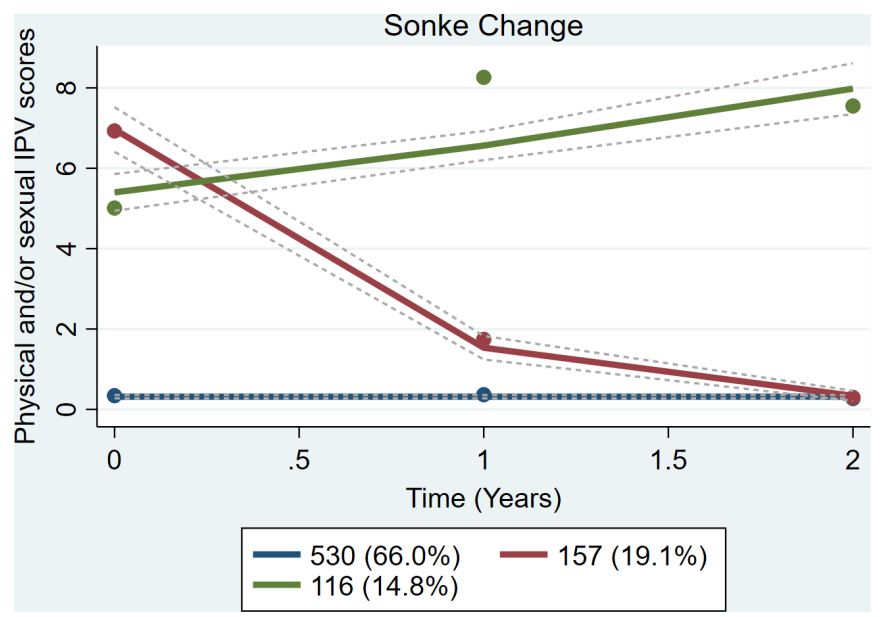

Figure 2 Trajectories of men's perpetration over time in the Sonke CHANGE trial. IPV, intimate partner violence.

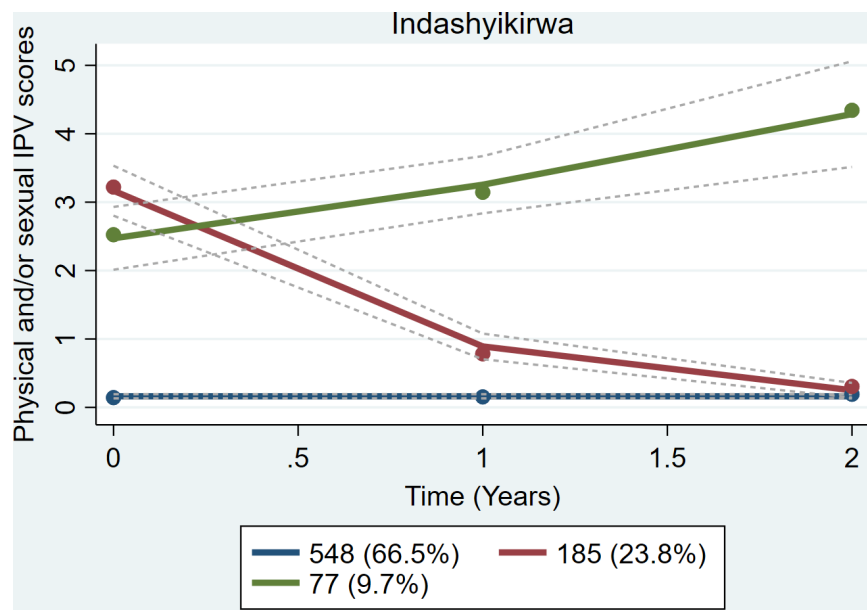

Figure 3 Trajectories of men's perpetration over time in the Indashyikirwa trial.IPV, intimate partner violence.

proportion of men in the high trajectories reported being beaten often or very often as a child (24.3\% low-flat; $37.3 \%$ high-reducing; $34.2 \%$ high-increase, $\mathrm{p}=0.002$ ). In all studies, a greater proportion of men in the high-start point trajectories reported witnessing their mother being beaten, and all were significantly higher than the low-flat trajectories.

Across all three studies, men in the high-start point trajectories reported poorer mental health and more substance misuse. In all three studies, a larger proportion of men reported depressive symptoms in the high-start point trajectories compared with the low-flat trajectory (eg, Indashyikirwa 6.7\%; $23.8 \% ; 29.2 \%, \mathrm{p}<0.001$ ). Similarly, in all three studies mean alcohol use scores were significantly higher among men in the high-start point trajectories, compared with those in the low-flat trajectories.

\section{Multinomial models comparing all three trajectories}

In all the multinomial models, there were broadly consistent patterns among men in the high-start point trajectories compared with men in the low-flat trajectories, including higher levels of poverty, more childhood traumas, worse mental health and more gender inequitable attitudes (tables 2A, 3A, 4A). In contrast, there were inconsistent findings regarding age and relationship status. Younger age was associated with being in the high-start point trajectories in Sonke CHANGE (highreducing: $R R R=0.96, p=0.03$; high-increasing $R R R=0.91$, $\mathrm{p}<0.001)$, and in Indashyikirwa, in the high-reducing ( $R R R=0.97, \mathrm{p}=0.028)$ groups compared with men in the low-flat trajectory, but there was no association with age in SS-CF. Additionally, in Sonke CHANGE, men in the high-increasing trajectory were less likely to have a partner they did not live with $(\mathrm{RRR}=0.32, \mathrm{p}<0.001)$ or not have a partner $(\mathrm{RRR}=0.56, \mathrm{p}=0.09)$ compared with the low-flat trajectory, but in the other two studies, relationship status was not associated with trajectory allocation.

In SS-CF and Sonke CHANGE, men in the highstart point trajectories were poorer than those in the 
low-flat trajectory. Specifically, in SS-CF, men in the highincreasing trajectory were more likely to report stealing because of hunger $(\mathrm{RRR}=2.49, \mathrm{p}=0.008)$, and were more likely to earn anything in the past month $(R R R=2.21$, $\mathrm{p}=0.023)$ than those in the low-flat trajectory. Men in SS-CF in the high-increasing trajectory reported less shame about lack of work ( $R R R=0.87, p=0.033)$ than those in the low-flat trajectory. In Sonke CHANGE, stealing in the past month because of hunger was more likely in the high-reducing trajectory $(\mathrm{RRR}=2.42, \mathrm{p}<0.001)$ and highincreasing trajectory $(\mathrm{RRR}=1.78, \mathrm{p}=0.019)$ compared with the low-flat trajectory. There was no indication of an association between poverty and trajectory allocation in Indashyikirwa.

In all studies, more childhood trauma was associated with allocation to high-start point trajectories. In SS-CF, men in the high-reducing trajectory $(R R R=1.07$, $\mathrm{p}=0.035)$, and in Sonke CHANGE in the high-reducing trajectory $(\mathrm{RRR}=1.08, \mathrm{p}<0.001)$ and high-increasing trajectory $(R R R=1.06, p=0.012)$ reported more childhood trauma than the low-flat trajectory. In Indashyikirwa and Sonke CHANGE, men in the high-start point trajectories reported witnessing their mother being beaten (Indashyikirwa high-reducing $R R R=1.62, \mathrm{p}=0.009$; high-increasing $\mathrm{RRR}=1.86, \mathrm{p}=0.021$; Sonke CHANGE high-increasing $\mathrm{RRR}=1.77, \mathrm{p}=0.03$ ) than men in the low-flat trajectories.

Depressive symptoms were associated with being in the high-start trajectories in all three studies. This was the case for men in SS-CF in the high-reducing $(\mathrm{RRR}=3.53$, $\mathrm{p}=0.002)$, and in CHANGE for the high-reducing $(R R R=2.71, p<0.001)$ and high-increasing $(R R R=1.90$, $\mathrm{p}=0.011$ ), and in Indashyikirwa both high-reducing $(\mathrm{RRR}=3.85, \mathrm{p}<0.001)$ and high-increasing $(\mathrm{RRR}=4.11$, $\mathrm{p}<0.001)$ trajectories, compared with the low-flat trajectories.

Gender inequitable attitudes were associated with the high-start trajectories in all three studies. In SS-CF compared with low-flat, acceptability of violence was associated with the high-reducing $(\mathrm{RRR}=2.97, \mathrm{p}=0.003)$ and high-increasing $(\mathrm{RRR}=2.88, \mathrm{p}=0.002)$ trajectories and in Sonke CHANGE with the high-reducing $(\mathrm{RRR}=1.53$, $\mathrm{p}=0.037)$ and high-increasing ( $R R R=1.84, \quad \mathrm{p}=0.007)$ trajectories. Similarly, in Indashyikirwa, supportive attitudes towards IPV were associated with being in the high-reducing $(\mathrm{RRR}=1.99, \mathrm{p}<0.001)$ and high-increasing $(\mathrm{RRR}=3.48, \mathrm{p}<0.001)$ trajectories compared with the lowflat trajectory.

\section{Comparing men in high-increasing trajectories to those in high-reducing trajectories}

In multivariable multinomial models comparing those in the high-increasing trajectories to those in the highreducing trajectories, there was variation by study. In SS-CF, men in the reducing trajectory were significantly more depressed ( $R R R=3.06, p=0.011)$ at baseline compared with the high-increasing trajectory (table 2B).

In Sonke CHANGE (table 3B), those in the highreducing trajectory compared the high-increasing trajectory were older $(\mathrm{RRR}=1.05, \mathrm{p}=0.06)$, were more likely to have a partner they did not live with $(R R R=2.14$, $\mathrm{p}=0.011$ ), were marginally more likely to report they had not earnt in the past month $(\mathrm{RRR}=0.56, \mathrm{p}=0.11)$, although they had worked in the past 3 months $(\mathrm{RRR}=1.85, \mathrm{p}=0.036)$. Men in the high-reducing trajectory were also less likely to have witnessed abuse of their mother $(\mathrm{RRR}=0.53, \mathrm{p}=0.021)$, and were marginally more likely to report depression $(\mathrm{RRR}=1.49, \mathrm{p}=0.131)$, than those in the high-increasing trajectory.

Finally, in Indashyikirwa, those in the high-reducing trajectory were younger $(\mathrm{RRR}=0.96, \mathrm{p}=0.076)$, and marginally less accepting of IPV attitudes ( $R R R=0.62$, $\mathrm{p}=0.111$ ) than those in the high-increasing trajectory (table 4B).

\section{DISCUSSION}

Our analysis of the trajectories of men's IPV perpetration in three African IPV prevention trials provides an opportunity to explore how men respond to interventions depending on their life experience and current context. In all three trials, there were remarkably consistent trajectories defined in the models: the low-flat trajectory (SS-CF 60\%; Sonke CHANGE 66\%; Indashyikirwa $67 \%$ ); a high start with large reduction trajectory comprising of approximately a fifth of men (SS-CF 19\%; Sonke CHANGE 19\%; Indashyikirwa 24\%); and, a high start with slight increase trajectory (SS/CF 21\%; Sonke CHANGE 15\%; Indashyikirwa 10\%). The use of scores, rather than binary measures to assess IPV, allows more nuance in capturing reduced violence perpetration as well as total cessation.

Across all three interventions, the interventions appeared to have the greatest benefit for about a fifth of men, with large decreases in the mean IPV scores reported after the intervention. These reductions were all seen at the first follow-up (12 months) and sustained at endline (24 months). This suggests that the benefit of interventions is seen relatively quickly, and that all those who benefit immediately have sustained benefit. However, this is partly likely to be a function of our data collection (every year), and modelling strategy with only three possible trajectories, and many more complex trajectories were likely, as described in qualitative research. ${ }^{14}$

By far the largest group of men, in all three studies, were those whose mean IPV perpetration scores started low and stayed low. It may be that some men did not directly benefit from the intervention in terms of changing their IPV perpetration. However, it is unclear how many of these men would have started low and increased over the study period. In other words, it is plausible that cases of men starting new IPV were averted. These men may have learnt relationship and communication skills, and also skills around gender equity and non-violence, which improved their relationships with partners, family members, and others, leading to wider, more positive improvements not captured around IPV. In this analysis, 
Table 2A Multinomial model assessing factors associated with trajectory membership Stepping Stones and Creating Futures multinomial models

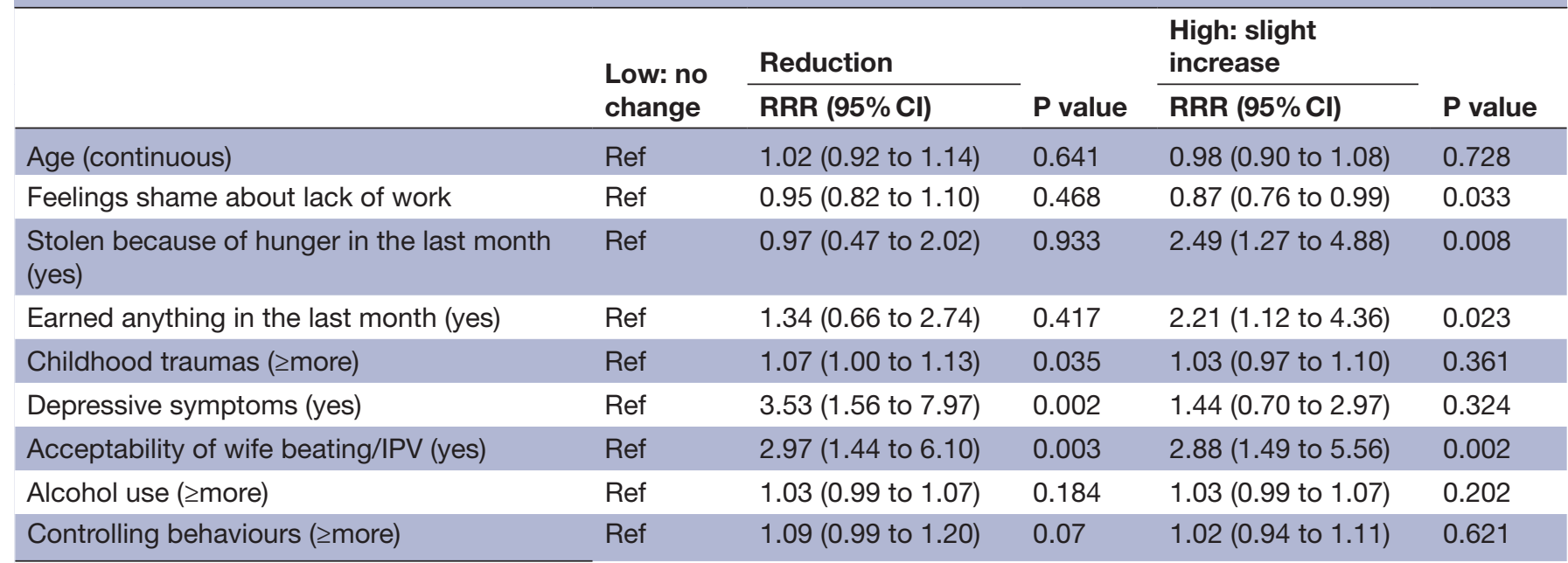

Model fit: $\mathrm{n}=286 ; \mathrm{p}<0.001 ; \mathrm{R}^{2}=0.15$.

IPV, intimate partner violence; RRR, relative risk ratio.

Table 2B Multinomial model assessing factors associated with different high trajectory membership in Stepping Stones and Creating Futures

\begin{tabular}{llll} 
& & High-reducing & \\
& High-increasing & RRR (95\% CI) & P value \\
\hline Age (continuous) & Ref & 1.05 (0.94 to 1.17) & 0.392 \\
Depressive symptoms (yes) & Ref & $2.49(1.14$ to 5.45) & 0.022 \\
\hline
\end{tabular}

Model fit: $n=105, p=0.01, R^{2}=0.08$.

$\mathrm{RRR}$, relative risk ratio.

we did not include the control arms, as the modelling approach did not allow us to compare differences by arm as in a trial analysis.

Mean scores for the high start with slight increase trajectory are likely a function of how we had to fit trajectories with limited time-points, using a maximum of three trajectories, and only linear functions possible. This trajectory likely reflects men who simply continued to perpetuate high levels of IPV, rather than an actual increase. It could also be, however, that some men experienced a backlash against their participation in an intervention where their authority and control over women was challenged. Because we do not assess the relationship between attendance and group allocation, we cannot be sure whether or not men attended the interventions. Regardless, it is important to consider potential negative implications for a small subset of men who do not derive the same benefits from intervention exposure.

Across the three studies, there were consistent patterns around the baseline factors associated with trajectory allocation between those in the flat-low trajectory and those in the trajectories that begin at high levels of perpetration. These reflect known factors with IPV perpetration, poverty, gender inequitable attitudes, poor mental health and childhood trauma. ${ }^{25} 30$ The one consistent factor in all multinomial models was men's acceptance of IPV which was always significantly higher in the two high-start point trajectories, compared with the flat-low trajectory. This suggests that men's own attitudes towards violence perpetration are indicative of their willingness to use IPV, and that a strong focus of designing interventions to prevent IPV should be on challenging the acceptability of IPV through transforming gender norms of individual participants.

Other factors associated with allocation to the highstart point IPV trajectories were less consistent across studies, and may have been contextually driven. Specifically, in Rwanda, no measures of poverty were associated with higher IPV perpetration, unlike South Africa where this was clearly the case. In Rwanda, the participants often farmed and had access to land and livestock in communities of similarly situated men, so grinding poverty and associated social stigma may have been less acutely experienced, while in South Africa, the men lived in urban informal settlements, with wide variations in access to work. This suggest that selection of risk factors for targeting in interventions needs to reflect clear contextual understandings of the drivers of IPV in any community. In all three of the interventions, a combination of these risk factors were targeted in the theories of change that guided intervention development.

Comparing men in the high-reducing trajectory and those in the high-slight increase trajectory, showed no consistent patterns across studies. In SS-CF, men in the 
Table 3A Multinomial model assessing factors associated with trajectory membership Sonke CHANGE trial multinomial models

\begin{tabular}{|c|c|c|c|c|c|}
\hline & \multirow[b]{2}{*}{ Flat-low } & \multirow{2}{*}{$\begin{array}{l}\text { High-reducing } \\
\text { RRR }(95 \% \mathrm{Cl}) \\
\end{array}$} & \multirow[b]{2}{*}{$P$ value } & \multirow{2}{*}{$\begin{array}{l}\text { High-increasing } \\
\text { RRR }(95 \% \mathrm{Cl})\end{array}$} & \multirow[b]{2}{*}{$P$ value } \\
\hline & & & & & \\
\hline Age (continuous) & Ref & $0.96(0.92$ to 1.00$)$ & 0.03 & $0.91(0.87$ to 0.95$)$ & $<0.001$ \\
\hline Living together & Ref & Ref & & Ref & \\
\hline Not living together & Ref & $0.69(0.44$ to 1.08$)$ & 0.103 & $0.32(0.19$ to 0.54$)$ & $<0.001$ \\
\hline $\begin{array}{l}\text { Stolen because of hunger in the last } \\
\text { month (yes) }\end{array}$ & Ref & 2.42 (1.58 to 3.70$)$ & $<0.001$ & 1.78 (1.10 to 2.89 ( & 0.019 \\
\hline Any earnings in the past month (yes) & Ref & 0.88 (0.65 to 1.59$)$ & 0.933 & 1.54 (0.83 to 2.86$)$ & 0.169 \\
\hline Childhood traumas ( $\geq$ more) & Ref & 1.08 (1.03 to 1.12$)$ & $<0.001$ & 1.06 (1.01 to 1.10$)$ & 0.012 \\
\hline Witnessed abuse of mother & Ref & 0.78 (0.48 to 1.29 & 0.335 & 1.77 (1.06 to 2.97 ) & 0.03 \\
\hline
\end{tabular}

Model fit: $n=784 ; p<0.001 ; R^{2}=0.13$.

IPV, intimate partner violence; RRR, relative risk ratio.

Table 3B Multinomial model assessing factors associated with different high trajectory membership in Sonke CHANGE trial

\begin{tabular}{|c|c|c|c|}
\hline & & High-reducing & \\
\hline & High-increasing & RRR (95\% Cl) & $P$ value \\
\hline Age (continuous) & Ref & $1.05(1.00$ to 1.11$)$ & 0.06 \\
\hline Relationship & & & \\
\hline Living together & Ref & Ref & \\
\hline Not living together & Ref & $2.14(1.19$ to 3.83$)$ & 0.011 \\
\hline No relationship & Ref & $1.07(0.47$ to 2.42$)$ & 0.869 \\
\hline Any earnings in the past month (yes) & Ref & $0.56(0.27$ to 1.14$)$ & 0.11 \\
\hline Worked in last 3 months (yes) & Ref & $1.85(1.04$ to 3.29$)$ & 0.036 \\
\hline Witnessed abuse of mother & Ref & $0.53(0.31$ to 0.91$)$ & 0.021 \\
\hline Depressive symptoms (yes) & Ref & $1.49(0.89$ to 2.50$)$ & 0.131 \\
\hline
\end{tabular}

Model fit: $n=264 ; p<0.01 ; R^{2}=0.06$.

$\mathrm{RRR}$, relative risk ratio.

high-reducing trajectory were more depressed compared with men in the high-increasing trajectory. In Sonke CHANGE, there was an indication these men (highreducing trajectory) similarly had more depression than men in the high-increasing trajectory. Qualitative data from SS-CF suggested that the intervention with intensive, group-based sessions over many weeks, created spaces for men to speak about their vulnerabilities for the first-time, including around lack of work, and that through the intervention, men started to realise this was a structural constraint, rather than a personal failing. ${ }^{31}$ In addition, men in the intervention in SS-CF started working more by end-line compared with the control arm. ${ }^{19}$ It could be that because SS-CF provided a therapeutic group space, and supporting young men to work more, SS-CF helped reduce these symptoms of depression, enabling men to reduce their self-blame and in turn reduce their use of IPV. The differences between the high-reducing trajectory, and high-increasing trajectory, may be linked to the different drivers of depression, and the extent to which depression was a marker of more entrenched mental health challenges, or driven by contextual factors. More entrenched mental health may have emerged through exceedingly harsh treatment in childhood, leading to entrenched patterns of disassociation and lack of empathy as a coping strategy, ${ }^{32}$ and markers of psychopathology are strongly associated with violence perpetration. ${ }^{33}$ It could therefore be, the lighttouch therapeutic space that SS-CF created, worked to ameliorate men's symptoms of depression where they were driven by current contexts (ie, poverty and sense of failure), elevating their self-esteem, but where underpinned by long-term trauma and abuse, this intervention could not impact on men. As such, it may be that those with the most entrenched issues, may need more direct therapeutic intervention. 
Table 4A Multinomial model assessing factors associated with trajectory membership Indashyikirwa trial multinomial models

\begin{tabular}{|c|c|c|c|c|c|}
\hline & \multirow[b]{2}{*}{ Flat-low } & \multirow{2}{*}{$\begin{array}{l}\text { High-reducing } \\
\text { RRR }(95 \% \text { Cl) }\end{array}$} & \multirow[b]{2}{*}{$P$ value } & \multirow{2}{*}{$\begin{array}{l}\text { High-increasing } \\
\text { RRR }(95 \% \mathrm{Cl})\end{array}$} & \multirow[b]{2}{*}{$P$ value } \\
\hline & & & & & \\
\hline Age (continuous) & ref & $0.97(0.95$ to 1.00$)$ & 0.028 & $1.01(0.97$ to 1.05$)$ & 0.643 \\
\hline Witnessed abuse of mother & ref & 1.62 (1.13 to 2.34$)$ & 0.009 & $1.86(1.10$ to 3.15$)$ & 0.021 \\
\hline Alcohol use ( $\geq$ more) & ref & 1.20 (1.09 to 1.31$)$ & $<0.001$ & $1.14(1.00$ to 1.29$)$ & 0.05 \\
\hline Depressive symptoms (yes) & ref & 3.85 (2.31 to 6.41$)$ & $<0.001$ & $4.11(2.15$ to 7.85$)$ & $<0.001$ \\
\hline Acceptability of wife beating/IPV ( $\geq$ more) & ref & 1.99 (1.38 to 2.87$)$ & $<0.001$ & 3.48 (2.01 to 6.03$)$ & $<0.001$ \\
\hline
\end{tabular}

Model fit: $n=780 ; p<0.001 ; R^{2}=0.09$.

IPV, intimate partner violence; $R R R$, relative risk ratio.

Table 4B Multinomial model assessing factors associated with different high trajectory membership in Indashyikirwa trial

\begin{tabular}{llll}
\hline & & High-reducing \\
\cline { 3 - 4 } & High-increasing & RRR (95\% Cl) & $0.96(0.92$ to 1.00$)$ \\
Age (continuous) & Ref & Ref & $0.63(0.35$ to 1.11$)$
\end{tabular}

Model fit: $n=256 ; p=0.04 ; R^{2}=0.03$.

IPV, intimate partner violence; RRR, relative risk ratio.

In the Sonke CHANGE trial, men who reduced their violence perpetration were less likely to have witnessed their mother being beaten in childhood, compared with men in the high with slight increase trajectory. This could be a marker that the intervention worked among men who were relatively amenable to change, without deeply entrenched learnt behaviours. Social learning theory suggests that IPV is learnt through witnessing the behaviour, through the internalisation of attitudes supportive of the behaviour, and through positive reinforcement of the behaviour, ${ }^{34}$ which therefore requires substantial work on all three-components to enact change in behaviour. The Sonke CHANGE trial was a 'lighttouch' social norms intervention, with workshops lasting a maximum of 2 days. Thus, it may be that men were better placed to respond to them when they did not have to overcome socially learnt behaviours from childhood, with corresponding poor mental health and substance use, of witnessing such violence. This somewhat substantiates another analysis of the Sonke CHANGE trial, which suggested that it may have reduced men's violence among those who were moderately violent at baseline and more amenable to light-touch interventions, but had no impact on those most violent, with attendant overlapping issues of poor mental health and substance misuse. ${ }^{20}$

In Rwanda, men in the high-reducing trajectory were less accepting of IPV compared with the high with slight increase trajectory. The finding around attitudes towards IPV suggests that these men may have already been more amenable to change, and that IPV was partly driven by pressure from social norms to discipline their wives ${ }^{35}$ and that the intervention provided men with alternative strategies of communication. ${ }^{36} 37$

In Rwanda, younger married/cohabiting men were more likely to reduce their violence, while in the Sonke CHANGE trial, it was older men, and those not living with their partner who reduced their IPV perpetration. The contextual realities of Rwanda and South Africa may help explain what types of masculinities could change through interventions. In primarily rural Rwanda, young men were married/cohabiting and earlier on in their relationships, with less experience in conflict resolution and negotiating relationships, and the Indashyikirwa intervention provided skills around communication and conflict resolution, as well as supporting men to think about power in relationships. ${ }^{37}$ In contrast, the Sonke CHANGE trial was implemented in urban informal settlements in South Africa, where young men were continually required to prove their masculinity against contexts of violence and poverty. ${ }^{38}$ Older men in South Africa may have been more established in their masculine position, and so more easily able to change, as they were less influenced by other men's (and women's) perceptions about them as men. ${ }^{39}$ In addition, it may be that men in the Sonke CHANGE trial changed partners during this period, and it was in new relationships they could establish alternative, less violent patterns.

This study has several limitations. A major challenge was that the variables we assessed may not adequately capture the underlying latent variable driving group allocation (eg, masculinity) and this explains some discrepancies between the different models, as masculinities function differently in every context. In addition, our sample size in all three studies limited statistical power to detect differences between groups. This had a number of impacts, first, we only could fit three potential trajectories, while it is likely men responded to the interventions in many other ways, as such our trajectories were chosen on fit statistics and theoretical plausibility. Second, the small sample sizes, particularly in comparing the two high-start point trajectories, may have led to many variables being removed from the 
regression that were important in reality. We also looked at only baseline values of potential predictors of men's trajectory allocation, and some of these, such as depression, will have changed over time and possibly through participation in the intervention. While we had similar variables across the studies, there were differences in what was asked, meaning we could not compare the analyses directly. As we had only two or three time-points for each person, we could only fit linear models, and it is likely with more time-points we may see different trajectories (eg, quadratic), which violence decreasing and then increasing, or vice-versa.

The analysis of which men change through participation in IPV prevention interventions is an important starting point in seeking to provide more complex answers to prevention questions. We identified three groups of men with different responses to the interventions, and explored what baseline characteristics were predictive of group allocation. Returning to our original two questions: (i) who is predisposed to change? And, (ii) do different interventions lead to different responses? The analysis showed no consistent pattern across the three studies about which baseline factors were associated with men's propensity to change. However, the modelling suggests that there may be benefit in targeting interventions towards men who are more violent, but that not all men who are violent have the potential to benefit in the same ways. Key structural and contextual considerations around access to work, mental health and ability to make meaning of past life traumas may be required to attain reductions in IPV perpetration.

There was also no clear pattern about whether more intensive group-based interventions (SS-CF and Indashyikirwa) impacted different men compared with lighter touch social norms change interventions (Sonke CHANGE). There was some suggestion that this may have been the case, with SS-CF potentially working better with men where IPV was driven by depression and structural constraints, and Sonke CHANGE where IPV was driven by social norms. However, the analysis more broadly suggests that there may well not be any intervention that works 'best', but rather that it is important to match the intervention strategy to the population that is being targeted, understand how masculinities function in a given setting and are related to IPV perpetration, and consider who is able to respond to the intervention components. Including severity of IPV as an outcome, rather than considering it as yes/no variable can help explore harm reduction and benefits of programme even where complete cessation of violence does not occur.

More than one-third of the world's women report experiencing IPV in their lifetimes, and it is clear that working directly with women survivors of abuse will never reach global prevention goals. Skilful and effective work with men should be a key element of future IPV prevention, and such work can be tailored to unique contexts and situations by addressing the deeper questions around what works, for whom, and why. Continued exploratory research around these questions is critical for deepening our understanding of how to prevent IPV.

\section{Author affiliations}

${ }^{1}$ Gender and Health Research Unit, South African Medical Research Council, Pretoria, Gauteng, South Africa

${ }^{2}$ Centre for Rural Health, University of KwaZulu-Natal, Durban, South Africa

${ }^{3}$ School of Public Health, University of the Witwatersrand, Johannesburg, South Africa

${ }^{4}$ University of North Carolina, Chapel Hill, United States

${ }^{5}$ Office of the Excutive Scientist, South African Medical Research Council, Pretoria, South Africa

Contributors AG, KD, SM, EC, AH, RJ and NJC conceptualised the analysis. AG, $\mathrm{KD}, \mathrm{EC}, \mathrm{AH}, \mathrm{CN}$ and RJ collected the data. EC and SH led the statistical analysis. AG wrote the first draft of the paper. All authors contributed to the interpretation and revision of the paper, and approved submission.

Funding This study was funded by DFID-UK as part of the What Works to Prevent Violence against Women and Girls Programme. Funding was managed by the South African Medical Research Council.

Competing interests None declared.

Patient and public involvement Patients and/or the public were not involved in the design, or conduct, or reporting or dissemination plans of this research.

\section{Patient consent for publication Not required.}

Ethics approval For Stepping Stones and Creating Futures, ethical approval was received from the University of KwaZulu-Natal's Biomedical Research Ethics Committee and the South African Medical Research Council's Ethics Committee. For the Sonke CHANGE trial, ethical approval was received from the University of Witwatersrand ethics committee. In Rwanda, approval to undertake the study was obtained from the Rwandan National Ethics Committee and the National Institute of Statistics Rwanda, as well as the South African Medical Research Council's Ethics Committee.

Provenance and peer review Not commissioned; externally peer reviewed.

Data availability statement Data are available in a public, open access repository. All data are available at http://medat.samrc.ac.za/index.php/catalog/ WW.

Open access This is an open access article distributed in accordance with the Creative Commons Attribution Non Commercial (CC BY-NC 4.0) license, which permits others to distribute, remix, adapt, build upon this work non-commercially, and license their derivative works on different terms, provided the original work is properly cited, appropriate credit is given, any changes made indicated, and the use is non-commercial. See: http://creativecommons.org/licenses/by-nc/4.0/.

ORCID iD

Andrew Gibbs http://orcid.org/0000-0003-2812-5377

\section{REFERENCES}

1 Jewkes R, Flood M, Lang J. From work with men and boys to changes of social norms and reduction of inequities in gender relations: a conceptual shift in prevention of violence against women and girls. Lancet 2015;385:1580-9.

2 Dworkin SL, Treves-Kagan S, Lippman SA. Gender-transformative interventions to reduce HIV risks and violence with heterosexuallyactive men: a review of the global evidence. AIDS Behav 2013;17:2845-63.

3 Barker G, Ricardo C, Nascimento M. Engaging men and boys in changing gender-based inequity in health: evidence from programme interventions. Geneva: WHO, 2007.

4 Flood M. Work with men to end violence against women: a critical stocktake. Cult Health Sex 2015;17 Suppl 2:159-76.

5 Gibbs A, Vaughan C, Aggleton P. Beyond 'working with men and boys': (re)defining, challenging and transforming masculinities in sexuality and health programmes and policy. Cult Health Sex 2015;17:85-95.

6 Doyle K, Levtov RG, Barker G, et al. Gender-transformative Bandebereho couples' intervention to promote male engagement in reproductive and maternal health and violence prevention in Rwanda: findings from a randomized controlled trial. PLoS One 2018;13:e0192756. 
7 Ruane-McAteer E, Amin A, Hanratty J, et al. Interventions addressing men, masculinities and gender equality in sexual and reproductive health and rights: an evidence and gap map and systematic review of reviews. BMJ Glob Health 2019;4:e001634.

8 Jewkes R, Morrell R, Hearn J, et al. Hegemonic masculinity: combining theory and practice in gender interventions. Cult Health Sex 2015;17 Suppl 2:112-27.

9 Michau L, Horn J, Bank A, et al. Prevention of violence against women and girls: lessons from practice. Lancet 2015;385:1672-84.

10 Morrell R, Jewkes R, Lindegger G. Hegemonic masculinity/ masculinities in South Africa: culture, power, and gender politics. Men and Masculinities 2012;15:11-30.

11 Morrell R, Jewkes R, Lindegger G, et al. Hegemonic masculinity: reviewing the gendered analysis of men's power in South Africa. South African Review of Sociology 2013;44:3-21.

12 Connell R. Gender, health and theory: conceptualizing the issue, in local and world perspective. Soc Sci Med 2012;74:1675-83.

13 Jewkes R, Morrell R. Masculinity violence, and gender equality: using latent class analysis to investigate the origins and correlates of differences between men. Men and Masculinities 2017;1097184X:17696171.

14 Gibbs A, Jewkes R, Sikweyiya Y, et al. Reconstructing masculinity? A qualitative evaluation of the stepping stones and creating futures interventions in urban informal settlements in South Africa. Cult Health Sex 2015;17:208-22.

15 Gibbs A, Washington L, Willan S, et al. The Stepping Stones and Creating Futures intervention to prevent intimate partner violence and HIV-risk behaviours in Durban, South Africa: study protocol for a cluster randomized control trial, and baseline characteristics. BMC Public Health 2017;17:336.

16 Christofides NJ, Hatcher AM, Pino A, et al. A cluster randomised controlled trial to determine the effect of community mobilisation and advocacy on men's use of violence in periurban South Africa: study protocol. BMJ Open 2018;8:e017579.

17 Dunkle K, Stern E, Chatterji S, et al. Indashyikirwa programme to reduce intimate partner violence in Rwanda: report of findings from a cluster randomized control trial. Pretoria, South Africa: What Works, 2019.

18 Jones BL, Nagin DS. A note on a Stata plugin for estimating groupbased trajectory models. Sociol Methods Res 2013;42:608-13.

19 Gibbs A, Washington L, Abdelatif N, et al. Stepping Stones and Creating Futures intervention to prevent intimate partner violence among young people: cluster randomized controlled trial. J Adolesc Health 2020;66:323-35.

20 Christofides N, Hatcher AM, Rebombo D, et al. Effectiveness of a multi-level intervention to reduce men's perpetration of intimate partner violence: a cluster randomised controlled trial. Trials 2020.

21 Abramsky T, Devries K, Kiss L, et al. A community mobilisation intervention to prevent violence against women and reduce HIV/ AIDS risk in Kampala, Uganda (the Sasa! study): study protocol for a cluster randomised controlled trial. Trials 2012;13:1-22.

22 Abramsky T, Devries K, Kiss L, et al. Findings from the Sasa! study: a cluster randomized controlled trial to assess the impact of a community mobilization intervention to prevent violence against women and reduce HIV risk in Kampala, Uganda. BMC Med 2014:12:122.

23 Dunkle K, Stern E, Mclean L, et al. Impact of Indashyikirwa: an innovative programme to reduce intimate partner violence in rural Rwanda. South Africa: SAMRC, 2019.
24 WHO. Who multi-country study on women's health and domestic violence against women. Geneva: WHO, 2005.

25 Fulu E, Miedema S, Jewkes R, et al. Why do some men use violence against women and how can we prevent it? quantitative findings from the United nations Multi-country study on men and violence in Asia and the Pacific. Bangkok: UNDP, UNFPA, UN Women and UNV, 2013.

26 Coates J, Swindale A, Bilinsky P. Household food insecurity access scale (HFIAS) for measurement of food access: indicator guide. Washington, DC: Food and Nutrition Technical Assistance Project, Academy for Educational Development, 2007.

27 Saunders JB, Aasland OG, Babor TF, et al. Development of the Alcohol Use Disorders Identification Test (AUDIT): WHO Collaborative Project on Early Detection of Persons with Harmful Alcohol Consumption--II. Addiction 1993;88:791-804.

28 Radloff LS. The CES-D scale a self-report depression scale for research in the general population. Appl Psychol Meas 1977;1:385-401.

29 Bernstein DP, Stein JA, Newcomb MD, et al. Development and validation of a brief screening version of the childhood trauma questionnaire. Child Abuse Negl 2003;27:169-90.

30 Fleming PJ, McCleary-Sills J, Morton M, et al. Risk factors for men's lifetime perpetration of physical violence against intimate partners: results from the International men and gender equality survey (images) in eight countries. PLoS One 2015;10:e0118639.

31 Gibbs A, Myrttinen H, Washington L, et al. Constructing, reproducing and challenging masculinities in a participatory intervention in urban informal settlements in South Africa. Culture, health \& sexuality 2019:1-16.

32 Perry BD. The neurodevelopmental impact of violence in childhood. Textbook of child and adolescent forensic psychiatry 2001:221-38.

33 Jewkes R, Sikweyiya Y, Morrell R, et al. Gender inequitable masculinity and sexual entitlement in rape perpetration South Africa: findings of a cross-sectional study. PLoS One 2011;6:e29590.

34 Akers R. Social learning and social structure: A general theory of crime and deviance. Routledge, 2017.

35 Stern E, Gibbs A, Willan S, et al. 'When you talk to someone in a bad way or always put her under pressure, it is actually worse than beating her': conceptions and experiences of emotional intimate partner violence in Rwanda and South Africa. PLoS One 2019;14:e0 225121-e21.

36 McLean L, Heise LL, Stern EA. Shifting and transforming genderinequitable beliefs, behaviours and norms in intimate partnerships: the Indashyikirwa couples programme in Rwanda. Cult Health Sex 2019:1-18.

37 Stern E, Heise L. Sexual coercion, consent and negotiation: processes of change amongst couples participating in the Indashyikirwa programme in Rwanda. Cult Health Sex 2019;21:867-82.

38 Gibbs A, Sikweyiya Y, Jewkes R. 'Men value their dignity': securing respect and identity construction in urban informal settlements in South Africa. Glob Health Action 2014;7:23676-10.

39 Hatcher AM, Colvin CJ, Ndlovu N, et al. Intimate partner violence among rural South African men: alcohol use, sexual decision-making, and partner communication. Cult Health Sex 2014;16:1023-39. 